\title{
Prácticas extracurriculares en el grado en Periodismo: rutinas profesionales, condicionantes y nivel de satisfacción de los alumnos
}

\author{
Media internships within the Journalism degree: \\ professional routines, conditioning factors and level of \\ satisfaction of the Spanish students
}

\author{
Bernardo Gómez-Calderón; Manuel García-Borrego; Manuel Fernández-Sande
}

Cómo citar este artículo:

Gómez-Calderón, Bernardo; García-Borrego, Manuel; Fernández-Sande, Manuel (2019). “Prácticas extracurriculares en el grado en Periodismo: rutinas profesionales, condicionantes y nivel de satisfacción de los alumnos". El profesional de la información, v. 28, n. 6, e280610.

https://doi.org/10.3145/epi.2019.nov.10

Artículo recibido el 12-02-2019 Aceptación definitiva: 19-09-2019

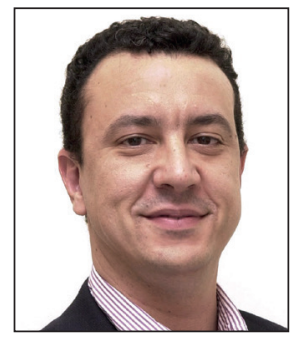

Bernardo Gómez-Calderón $\triangle$ https://orcid.org/0000-0002-9245-9251

Universidad de Málaga

Facultad de Ciencias de la Comunicación Campus de Teatinos

León Tolstoi, 4. 29010 Málaga, España bjgomez@uma.es

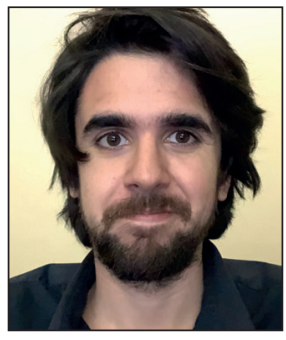

Manuel García-Borrego

https://orcid.org/0000-0001-6207-8741

Universidad de Málaga

Facultad de Ciencias de la Comunicación Campus de Teatinos

León Tolstoi, 4. 29010 Málaga, España

manoletus@uma.es

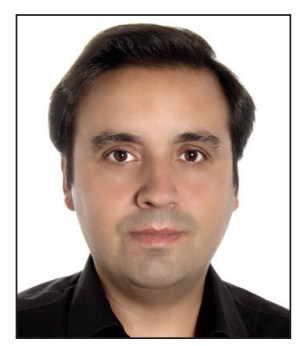

Manuel Fernández-Sande

https://orcid.org/0000-0002-0740-2630

Universidad Complutense de Madrid, Facultad de Ciencias de la Información Av. Complutense, 3.

28040 Madrid, España

manuel.fernandez@ucm.es

\section{Resumen}

El artículo analiza las condiciones en que se desarrollan las prácticas extracurriculares del grado en Periodismo, con el propósito de determinar las rutinas y los condicionantes más habituales a los que se ven sometidos los estudiantes y su nivel de satisfacción con la experiencia. A partir de un cuestionario respondido por alumnos de las universidades de Málaga y Complutense de Madrid ( $\mathrm{n}=210$ ), se concluye que el sistema de prácticas en Periodismo es asimétrico, más exigente según el tipo y tamaño de la empresa y la sección en que éstas se desarrollen; que la labor de los tutores es dispar, mostrándose mucho más implicado el responsable de empresa que el académico, apenas conocido por los alumnos; y que más de la mitad los estudiantes se ven obligados con frecuencia a firmar textos que les desagradan desde el punto de vista ético o profesional, entre otras constataciones de interés.

\section{Palabras clave}

Periodismo; Universidad; Grado en Periodismo; Prácticas en empresas; Rutinas profesionales; Medios de comunicación; Gabinetes; Condiciones laborales; Becarios; Estudiantes; Universidad de Málaga; Universidad Complutense de Madrid; España. 


\begin{abstract}
This article studies the conditions of extracurricular internships carried out by Spanish journalism students in order to describe their regular professional routines, the elements that most frequently determine their work and their level of satisfaction with the stay. A survey was conducted in the universities of Málaga and Complutense of Madrid ( $n=210$ ), whose results present an asymmetric internship system: among other findings, the training conditions depend on factors such as the type and size of the company or the section where the students are allocated; the supervision is dissimilar, with company tutors being much more dedicated than the academic ones, hardly known by the interns; and more than half of the students have been compelled to sign pieces they find unethical or barely professional.
\end{abstract}

\title{
Keywords
}

Journalism; University; Journalism degree; Internships; Professional routines; Media; Press offices; Working conditions; Trainees; Students; University of Málaga; Complutense University of Madrid; Spain.

\section{Introducción}

Las prácticas extracurriculares constituyen un componente generalmente asociado a la formación superior en Periodismo. A través de ellas los alumnos ingresan en una comunidad cuyos miembros originales comparten actitudes, valores y conocimientos que nutren su incipiente socialización profesional (Lave; Wenger, 1991; Gaunt, 1992; Splichal; Sparks, 1994; Preston, 2009; Gravengaard; Rimestad, 2014).

En todos los casos en que se encuentran institucionalizados, estos periodos se caracterizan por su naturaleza académica en lugar de laboral, y por desarrollarse en un contexto de precariedad: quienes se desempeñan como becarios carecen de cualquier tipo de cobertura, pese a que con frecuencia sustituyen a redactores a tiempo completo (Gollmitzer, 2014).

La duración de las prácticas varía: en Dinamarca son de año o año y medio y suelen emplazarse en el ecuador del grado (Gravengaard; Rimestad, 2014), mientras que en Suecia y Noruega abarcan solo seis meses (Willig, 2016). En Alemania existe el voluntariat, posterior a los estudios universitarios y escasamente remunerado, que puede prolongarse hasta dos años (Frölich; Holtzh-Bacha, 2009). En Canadá las prácticas son con frecuencia de carácter gratuito, aunque este aspecto difiere de unas provincias a otras (Salamon, 2015). En el resto de países anglosajones - singularmente, Reino Unido y Estados Unidos - no se encuentran institucionalizadas, de modo que es imposible describir con carácter general las condiciones bajo las que se desarrollan (Willig, 2016).

Junto con la precariedad y la escasa remuneración ${ }^{1}$, la otra característica común a todos los sistemas de prácticas parece ser su elevado nivel de exigencia: habitualmente los estudiantes desempeñan labores propias del personal contratado, y durante el mismo número de horas al día (Lamuedra, 2007; Standing, 2011; Segarra-Saavedra; Páramo-Galdón; Puebla-Martínez, 2012; García-Borrego; Roses-Campos, 2016).

En España las prácticas se regulan por el Real decreto 592/2014, de 11 de julio, que amplía la primera normativa aprobada al respecto, el Real decreto 1497/1981, de 19 de junio, y establece los preceptos mínimos a partir de los cuales las universidades desarrollan posteriormente sus convenios específicos. De modo genérico, se realizan durante los dos últimos años de carrera, una vez superado el $50 \%$ de los créditos de la titulación. Con respecto a las condiciones de trabajo, el real decreto no estipula horarios ni remuneración concretos.

Desde el punto de vista académico, el interés por las prácticas extracurriculares en Periodismo ha sido hasta ahora solo intermitente en nuestro país. El primer estudio de relevancia (Cantalapiedra; Coca; Bezunartea, 2000), circunscrito al País Vasco, llamaba la atención sobre una posible ruptura del mercado laboral, ya que la "sobreabundancia" de estudiantes en las redacciones provocaba que, en determinados medios, estos sobrepasaran en número a los periodistas de plantilla. Los alumnos desempeñaban la función de sustitutos, integrando un contingente de mano de obra "no ya barata, sino regalada" (Cantalapiedra; Coca; Bezunartea, 2000, p. 3).

Algún tiempo después, Blanco-Castilla (2005) en una encuesta a 40 becarios de la Universidad de Málaga constataba que el $60 \%$ superaba en tres horas o más a día el horario que figuraba en el convenio con la empresa. Las prácticas eran remuneradas sólo en el $65 \%$ de los casos, pese a lo cual los alumnos mostraban un elevado índice de satisfacción con ellas (90\% de puntuaciones positivas), pues consideraban que eran la mejor forma de acceder a un futuro contrato.

Por su parte, Lamuedra (2007) en un análisis de 100 memorias de estudiantes de la Universidad Carlos III de Madrid que realizaron prácticas curriculares - distintas de las extracurriculares aquí abordadas-, ahondaba también en el excesivo número de tareas que comportaban y en lo prolongado de las jornadas.

Esta línea la retomarían años más tarde Ortiz-Sobrino, Peinado-Miguel y Zapata-Palacios (2014) y Pérez-Serrano, Rodríguez-Barba y Rodríguez-Pallares (2015), quienes a través del estudio de 1.396 ofertas de prácticas de la Universidad Complutense de Madrid, calculaban la

Los índices globales de satisfacción quedan por debajo del aprobado en las dos universidades analizadas 
jornada media de los becarios en 5,3 horas y su retribución en 223 euros mensuales, para acabar reclamando una mejora de la tutela y los sistemas de control de calidad, con el objeto de evitar sustituciones encubiertas de redactores de plantilla y "trabajo no remunerado disfrazado de convenio con un centro educativo" (Pérez-Serrano; Rodríguez-Barba, Rodríguez-Pallares, 2015, p. 211).

Contribuciones recientes en este terreno son las de García-Borrego y Roses-Campos (2016) y García-Borrego, Roses-Campos y Farias-Batlle (2017). En una encuesta a los becarios de los principales medios de comunicación de Málaga (n=33), estos autores recogían que el 63,7\% percibía entre 301 y 400 euros al mes, frente a un 6,1\% que no recibía ningún tipo de remuneración -estos últimos sujetos a convenios distintos del de la Universidad de Málaga-, por unas jornadas que promediaban 6,6 horas. Los niveles de satisfacción generales eran considerablemente altos (7,64 puntos sobre 10), pese a que las condiciones no fuesen consideradas óptimas, ya que un $30 \%$ de los encuestados les otorgaba una calificación inferior a 5, y el promedio general no pasaba de 5,33.

A propósito de la experiencia de los estudiantes en gabinetes, un terreno hasta ahora poco explorado, García-Borrego y Gómez-Calderón (2018) concluían, de acuerdo con un cuestionario respondido por estudiantes de la UMA, que los alumnos que desarrollan sus prácticas en departamentos de comunicación les dedican menos horas al día que sus compañeros de medios, elaboran menos piezas y disponen de más tiempo para cada una de ellas. En consecuencia, su satisfacción es mayor en casi todos los aspectos contemplados por los autores: remuneración, estabilidad, horario, carga de trabajo, política de la empresa y relación con los compañeros o los superiores.

En otras áreas geográficas tampoco abundan los análisis sobre nuestro objeto de estudio, como advierten Gollmitzer (2014) y Salamon (2015). No obstante, la investigación se halla por lo general más evolucionada y, junto a acercamientos de propósito descriptivo, hay otros que abordan el modo en que los estudiantes interiorizan las rutinas profesionales: así, Gravengaard y Rimestad (2014) plantean que buena parte del proceso de enseñanza-aprendizaje se produce de forma tácita, asimilando los futuros periodistas las pautas de trabajo de modo implícito.

Otra línea de investigación, cultivada sobre todo en los países del norte de Europa, se ocupa de los principios, aspiraciones y expectativas de los alumnos que disfrutan de periodos de prácticas. Parece que a medida que éstas avanzan, los becarios pasan de una concepción idealista a otra más pragmática de la profesión (Bjørnsen; Hovden; Ottosen, 2007).

Teniendo en cuenta todo lo anterior, en este trabajo se decidió profundizar en las condiciones en que los estudiantes del grado en Periodismo desarrollan sus prácticas en nuestro país. Como primer objetivo (01) se planteó determinar a qué rutinas profesionales se ven sometidos de modo más frecuente, más allá del consabido eje jornada-remuneración (imposible de obviar pero susceptible de ser matizado); a continuación (O2) interesaba fijar en qué medida los alumnos se ven obligados, durante este periodo formativo, a realizar tareas que contravienen sus principios y valores, y si padecen las cortapisas que habitualmente se denuncian como consustanciales al ejercicio de la profesión periodística, algo sobre lo que se carecía de referencias en la bibliografía existente; y en tercer lugar, se consideró oportuno indagar sobre su nivel de satisfacción con las prácticas y el modo en que éstas influyen en sus expectativas laborales a medio plazo (O3). La investigación tendría una dimensión descriptiva, pero también comparativa, ya que se optó por sondear a estudiantes de dos universidades, la de Málaga y la Complutense de Madrid, de manera que, por primera vez en relación con nuestro objeto de estudio, pudieran establecerse similitudes y divergencias desde el punto de vista geográfico (O4).

\section{Metodología}

Para recabar la información que permitiera alcanzar los objetivos propuestos, consideramos que el método de investigación más apropiado era la encuesta a través de cuestionario. A partir de la bibliografía existente ${ }^{2}$ se elaboraron 29 preguntas que quedaron distribuidas en tres bloques temáticos:

- información de registro,

- rutinas profesionales, y

- nivel de satisfacción.

Las modalidades de respuesta eran variadas, abiertas o cerradas según los casos, y especialmente en la última parte se apoyaban en escalas Likert.

El cuestionario se suministró vía correo electrónico en dos oleadas, los días 15 y 22 de octubre de 2018, a los estudiantes de las tres últimas promociones de Periodismo de las universidades de Málaga (345) y Complutense de Madrid (1.672) . La elección de estas dos instituciones vino determinada por la adscripción laboral de los autores; pero se consideró que las diferencias entre UMA y UCM por lo que a número de alumnos ${ }^{4}$, antigüedad de los estudios y ubicación geográfica se refiere, enriquecería los resultados y los dotaría de mayor entidad.

De los estudiantes que componían el universo, 89 en el caso de UMA y 630 en el de UCM ${ }^{5}$ habían realizado prácticas durante el curso 2017-2018, que fue el periodo elegido para nuestro análisis. Retrotraernos más en el tiempo habría puesto 
en riesgo, creímos, la fidelidad del recuerdo esperable en un estudio sobre acontecimientos y percepciones. Después de un mes en que el cuestionario permaneció disponible online, se alcanzó la cifra de 210 respuestas, distribuidas entre 59 de la UMA y 161 de la UCM.

En la última fase del estudio, los datos de la matriz fueron sometidos a diversos procedimientos de análisis estadístico, principalmente descriptivo pero también bivariable (prueba t de Student) y multivariable, donde se consideró oportuno, por medio de regresiones lineales múltiples.

\section{Resultados}

\subsection{Características del sistema de prácticas}

El primer bloque del cuestionario permite establecer ya las propiedades fundamentales del sistema de prácticas en las dos universidades elegidas: la mayoría se realiza en medios de comunicación (70,2\%), en detrimento de los gabinetes, cuyo índice en cualquier caso no resulta desdeñable; y por ellas, los estudiantes perciben una remuneración media cercana a los 300 euros mensuales $(298,64)$, si bien en este terreno la dispersión de los registros es elevada, ya que la retribución iguala o supera el medio millar de euros en el 10,8\% de los casos, al tiempo que hay un 10,5\% de encuestados que no recibe nada en pago por su trabajo.

Casi la mitad de las respuestas obtenidas procede de alumnos que se encuentran cursando el último año de carrera y, en cuanto al tamaño de las empresas colaboradoras, las proporciones se distribuyen casi a partes iguales entre medianas y pequeñas $(40,4 \%-41,8 \%$ de incidencias).

Tabla 1. Descripción de la muestra

\begin{tabular}{|l|l|l|l|l|}
\hline Sexo & Mujer: $65,7 \%$ & Hombre: $34,3 \%$ & \\
\hline Edad & Media: 22,9 & DT: 1,984 & Mínimo: 20 \\
\hline Universidad & UCM: $71,9 \%$ & UMA: $28,1 \%$ & \\
\hline Curso & Tercero: $22,4 \%$ & Cuarto: $45,7 \%$ & Créditos sueltos: $15,2 \%$ \\
\hline Empresa & Medio: $70,2 \%$ & Gabinete: $29,8 \%$ & \\
\hline Tamaño & Pequeña $(1-10): 40,4 \%$ & Mediana $(11-99): 41,8 \%$ & Grande $(+100): 17,8 \%$ \\
\hline En verano acabada: $16,7 \%$ \\
\hline Remuneración & Sí: $67,6 \%$ & No: $32,4 \%$ & & \\
\hline
\end{tabular}

En este estadio no es posible diferenciar de modo significativo a los estudiantes de la UMA de los de la UCM, salvo en un aspecto: el de la remuneración (tabla 2). Ni por horas dedicadas al día - en torno a seis ${ }^{6}$ - ni por número de piezas elaboradas - unas cuatro- varían sustancialmente las condiciones de unos y otros; sin embargo, la prueba t de Student arroja un resultado significativo entre las medias de las dos universidades por lo que a la retribución se refiere: $t(152,7)=5,782(p<0,001)$.

Estos datos concuerdan con lo establecido por las respectivas normativas de prácticas, ya que la UMA es más garantista en el aspecto económico, al asegurar a todos los alumnos una dotación mínima de 360 euros mensuales, mientras que la UCM solo estipula el derecho a percibir la aportación de la entidad colaboradora, en concepto de bolsa o ayuda al estudio, sin determinar máximos ni mínimos.

Tabla 2. Condiciones de trabajo

\begin{tabular}{|l|c|c|c|}
\hline Remuneración & $268,0 €(\mathrm{DT}=153,0 €)$ & $375,7 €(\mathrm{DT}=104,1 €)$ & $\mathrm{t}(152,7)=5,782^{*}$ \\
\hline Horas dedicadas al día & $5 \mathrm{~h} 45 \mathrm{~m}(\mathrm{DT}=1 \mathrm{~h} \mathrm{32 \textrm {m } )}$ & $6 \mathrm{~h} 06 \mathrm{~m}(\mathrm{DT}=1 \mathrm{~h} \mathrm{40m)}$ & - \\
\hline Piezas realizadas al día & $3,9(\mathrm{DT}=3,33)$ & $4,1(\mathrm{DT}=3,69)$ & - \\
\hline
\end{tabular}

$* \mathrm{p}<0,001$

La diferencia entre las remuneraciones estándar de una y otra universidad supera los cien euros, ubicándose el grupo más numeroso de la UCM en el tramo que va de 251 a 300 (34,9\%), mientras que los estudiantes de la UMA se sitúan, en el $55,2 \%$ de los casos, entre los 350 y los 400 euros mensuales (gráfico 1).

Habida cuenta de que la adscripción geográfica no resultaba determinante desde el punto de vista estadístico para explicar la duración de la jornada de los becarios, se recurrió al análisis multivaria-

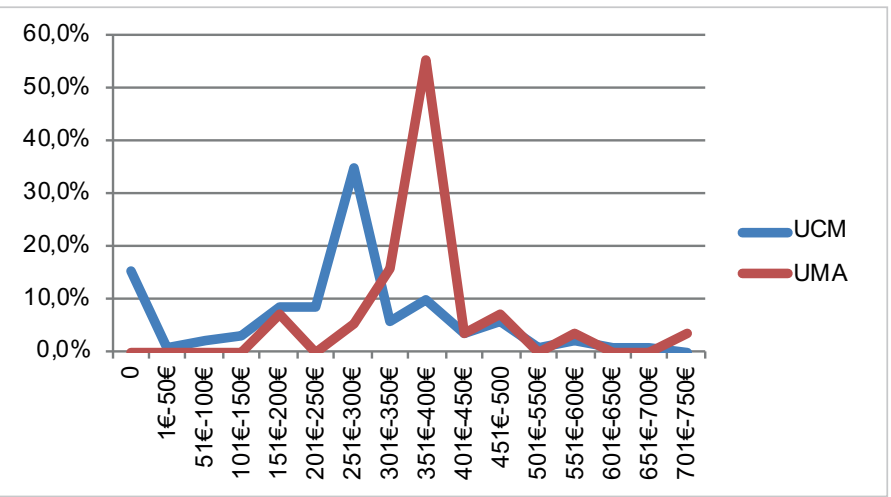

Gráfico 1. Rangos de remuneración por universidades (en porcentaje de casos) 
ble para comprobar si existían predictores de otra naturaleza. $Y$ en efecto, tal y como queda recogido en la tabla 3, la regresión lineal múltiple desveló que factores como trabajar en un medio (frente a los gabinetes de comunicación), desarrollar las prácticas en periodo estival, hacerlo en una empresa mediana o grande y encuadrarse en la sección de Local, conllevan por lo general jornadas más prolongadas, con rangos de significación ( $p$ ) en ningún caso superiores a 0,017. Por el contrario, estar adscrito al área de Deportes implica menos horas de dedicación al día. La tasa de varianza explicada a través de esta regresión ascendió al 25,7\%, lo cual en ciencias sociales puede considerarse estimable (Igartua-Perosanz, 2006).

Tabla 3. Coeficientes de regresión para la variable "Horas dedicadas al día"

\begin{tabular}{|c|c|c|c|c|}
\hline \multirow{2}{*}{ Modelo } & \multicolumn{2}{|c|}{ Coeficientes no estandarizados } & \multirow{2}{*}{$\begin{array}{c}\begin{array}{c}\text { Coeficientes } \\
\text { estandarizados }\end{array} \\
\text { Beta }\end{array}$} & \multirow[b]{2}{*}{ Sig. } \\
\hline & B & Error estándar & & \\
\hline (Constante) & 2,926 & 1,228 & & 0,018 \\
\hline Prácticas en un medio informativo & 1,082 & 0,304 & 0,307 & 0,000 \\
\hline Tamaño de la empresa & 0,396 & 0,156 & 0,195 & 0,012 \\
\hline Prácticas en verano & 0,696 & 0,227 & 0,203 & 0,003 \\
\hline Prácticas en la sección de Local & 0,672 & 0,318 & 0,176 & 0,036 \\
\hline Prácticas en la sección de Deportes & $-0,806$ & 0,362 & $-0,151$ & 0,027 \\
\hline$r^{2}$ ajustado & 0,257 & - & - & - \\
\hline Número de observaciones & 195 & - & - & - \\
\hline$F(17)$ & 4,938 & - & - & 0,000 \\
\hline
\end{tabular}

\subsection{Rutinas profesionales}

Abordando ya rutinas sobre las que no se habían hallado referencias en estudios previos, el cuestionario planteaba a los estudiantes con qué asiduidad recurrieron durante su periodo de prácticas al tutor de empresa -integrante de la plantilla del medio o gabinete- y al tutor académico - designado por la universidad-. Ambas figuras se encuentran previstas en el Real decreto 592/2014, donde se les atribuye una labor orientadora; sin embargo, su implicación en el desempeño de los futuros periodistas no puede ser más dispar a tenor de los resultados obtenidos.

Es cierto que existe un grupo de encuestados que declara no haber conocido al responsable que tenía asignado dentro de la empresa en la que trabajó (11,9\% en la UMA y $8,3 \%$ en la UCM) (gráficos 2 y 3); también que más de un $20 \%$ asegura no haberse dirigido nunca a él. Aun así, los dos tercios de alumnos restantes sí que le planteaban dudas o problemas con diversa regularidad.

Pero llama la atención el caso del tutor académico, cuya figura casi no aflora en las respuestas al cuestionario: un 46,8\% de estudiantes de la Complutense no conocía su identidad, y tampoco el 44,1\% de los de Málaga; y solo afirma haber recurrido a él un 3,8\% de los encuestados en Madrid (10,2\% en el caso de la UMA) (gráficos 4 y 5 ).

En consecuencia, la tarea de orientación profesional parece claramente decantada del lado del responsable de la empresa, sin que la adscripción territorial influya de modo apreciable en los resultados obtenidos.
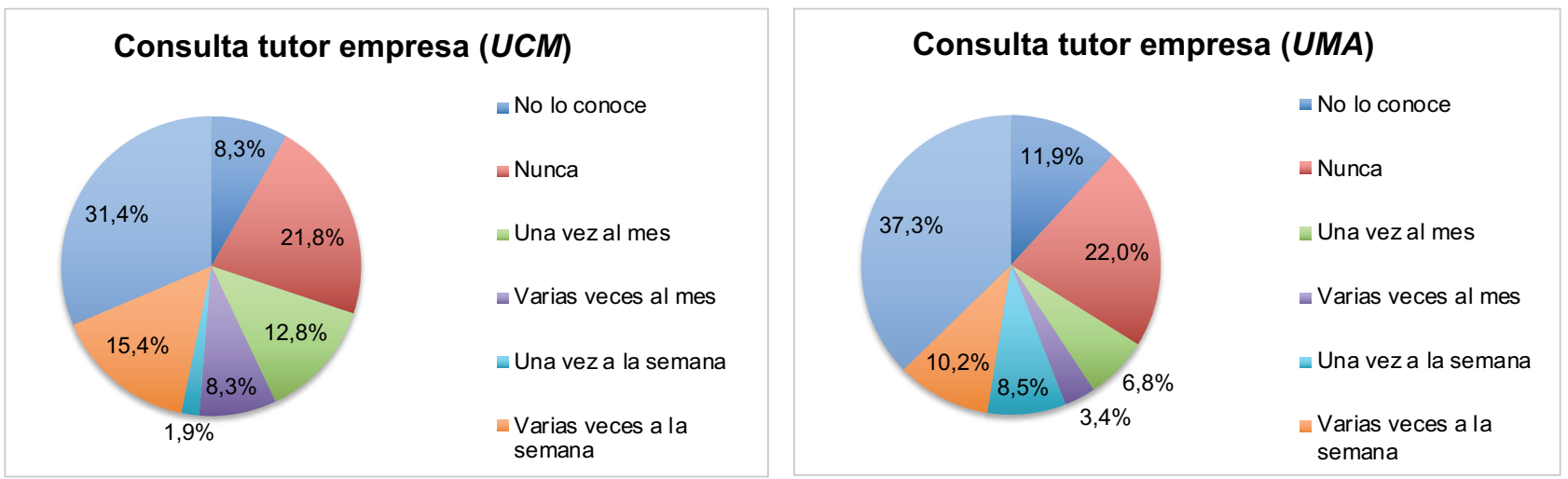

Gráficos 2-3. Frecuencia de consulta al tutor de empresa 

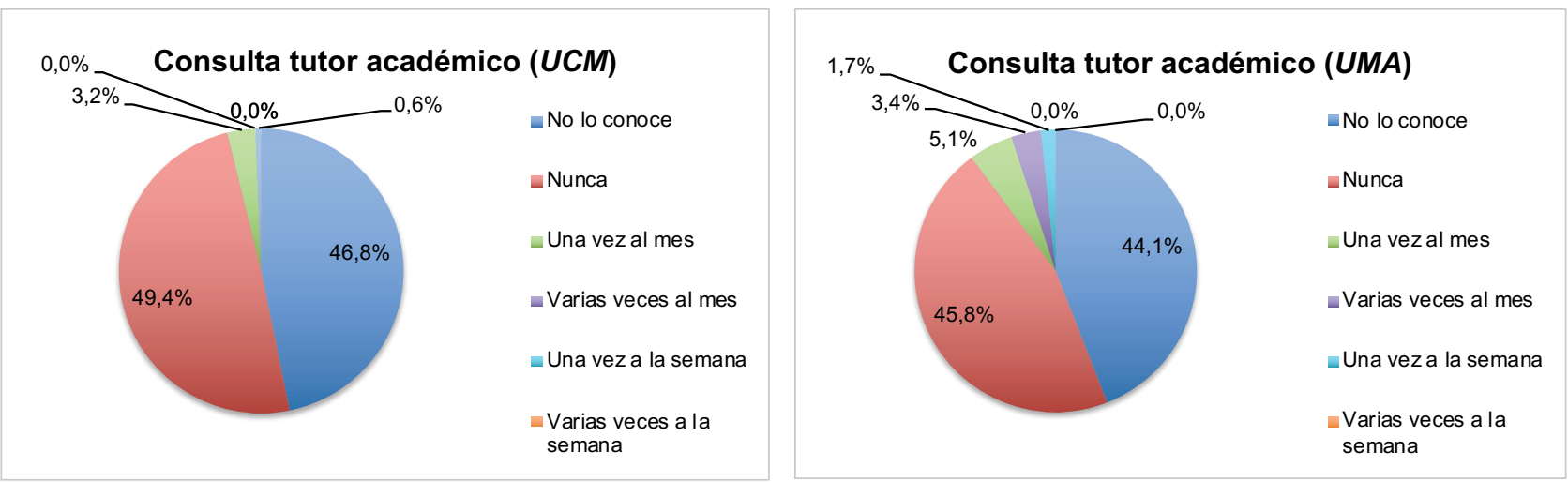

Gráficos 4-5. Frecuencia de consulta al tutor académico

Más allá de las consultas concretas que puedan plantearse, las prácticas parecen estar presididas por una dinámica colaborativa. Con asiduidad se da a los becarios la opción de proponer temas ("siempre" según un $25,8 \%$ de encuestados, y "habitualmente" hasta en un $41,1 \%$ de casos); y en mayor proporción incluso $(56,6 \%)$ se les ofrece la posibilidad de decidir el enfoque, o al menos, de dar su visión sobre el tratamiento del contenido que deben elaborar.

Otra rutina extendida, esta de repercusión directa en el proceso de aprendizaje de los alumnos, es la de corregir sus textos, algo que en el $43,5 \%$ de las empresas se realiza en todas los ocasiones y en el $33,5 \%$ se acomete con alguna frecuencia. La ausencia de supervisión de las tareas de los estudiantes constituye una circunstancia excepcional (4,3\% de respuestas).

Las razones por las que los responsables de los medios o gabinetes modifican las piezas elaboradas por los alumnos apuntan a ciertas carencias desde el punto de vista formativo, pues en el 51,5\% de las ocasiones afectan a cuestiones de estilo, ortografía y gramática. El resto de correcciones se refiere a aspectos de carácter periodístico, que quienes comienzan a ejercer la profesión es lógico que no dominen y necesiten aprender y perfeccionar, como la precisión de lo que narran (45,5\% de incidencias) o el empleo de recursos que atraigan la atención de la audiencia (25\%) -este último aspecto, no necesariamente positivo-. En un preocupante $8 \%$ de casos, los estudiantes denuncian la intevención de los editores para reducir la imparcialidad de sus textos.

La socialización profesional también acarrea en muchos casos experiencias poco edificantes. En el cuestionario se preguntaba a los alumnos por la realización de tareas que fueran en contra de sus valores deontológicos o profesionales; y aunque es cierto que muchos aseguran no haber firmado nunca piezas con cuyo contenido u orientación no estuvieran de acuerdo (algo más en la Complutense, 47,3\%, que en la UMA, 40,7\%), son mayoría quienes sí lo han hecho (52,4\%). Es más, hay un $18,7 \%$ de encuestados de la UCM para los que la colisión con sus principios éticos se producía siempre o habitualmente; en el caso de Málaga, el índice es algo inferior (12\%).

Cuando las tareas encargadas implicaban algún conflicto moral o profesional para los becarios, la respuesta de éstos variaba (gráfico 6). Un $21 \%$ se limitaba a acatar las directrices de la empresa, sin oponer resistencia; mientras que el resto adoptaba alguna de las siguientes estrategias, que podían combinarse o sucederse en función de la envergadura que adquiriera la disputa: argumentar su punto de vista (67\%), buscar el apoyo de los compañeros (39\%) o, de forma minoritaria, apelar a la cláusula de conciencia (8\%).

Los estudiantes que realizan prácticas perciben ya, pese a su socialización profesional solo incipiente, algunos de los condicionantes que definen el ejercicio del periodismo (cf. APM, 2018). De modo más acentuado les afectan el imperativo de ser

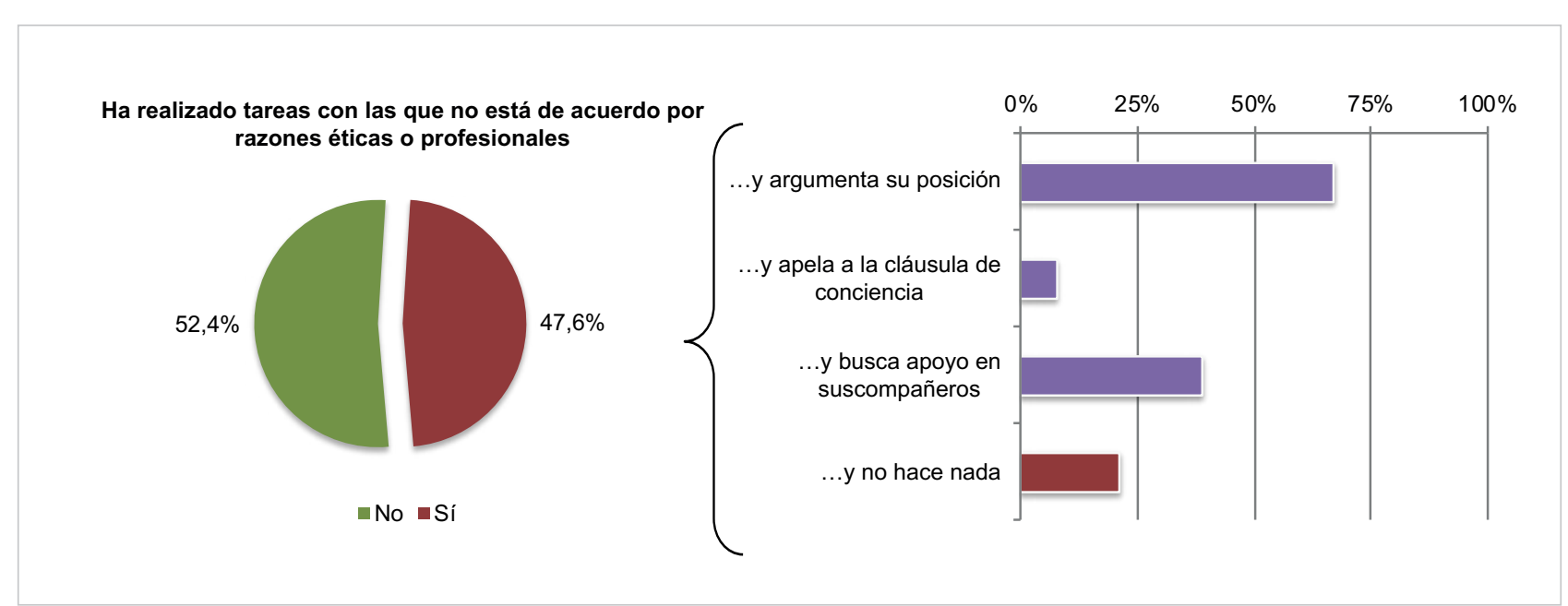

Gráfico 6. Respuesta ante conflictos de índole ética o profesional 
productivos (en una escala del 1 al 5, este factor obtiene puntuaciones de 2,78 en la UCM y 2,73 en la UMA) y la necesidad de captar el interés del público (2,77-2,34 de media, respectivamente). Otras circunstancias parecen repercutir de forma desigual en el día a día de los encuestados, como la presión de los jefes o el desconocimiento de los temas abordados (tabla 4).

En general, los condicionantes profesionales pesan más entre los alumnos de la Universidad de Málaga que entre los de la Complutense (así ocurre en seis de las ocho categorías contempladas), aunque el análisis bivariable solo arroja diferencias signiticativas $(p<0,001)$ en tres aspectos: la presión ejercida por las partes implicadas en la información, la falta de tiempo para llevar a cabo las tareas encomendadas - ambas mayores entre los estudiantes de la UMA- y la carencia de recursos técnicos - más acusada en el caso de la UCM-.

Tabla 4. Principales condicionantes percibidos en el ejercicio de la profesión (sobre 5 puntos)

\begin{tabular}{|c|c|c|c|}
\hline & & & \\
\hline & UCM & UMA & t de Student \\
\hline Condicionado por la presión de ser productivo & $2,78(\mathrm{DT}=1,42)$ & $2,73(\mathrm{DT}=1,23)$ & \\
\hline Condicionado por la necesidad de captar el interés de la audiencia & $2,77(\mathrm{DT}=1,36)$ & $2,34(\mathrm{DT}=1,35)$ & \\
\hline Condicionado por el desconocimiento del tema & $2,33(\mathrm{DT}=1,15)$ & $2,46(\mathrm{DT}=1,12)$ & \\
\hline Condicionado por la presión de los jefes & $2,21(\mathrm{DT}=1,42)$ & $2,44(\mathrm{DT}=1,45)$ & \\
\hline Condicionado por la falta de tiempo & $2,09(\mathrm{DT}=1,07)$ & $2,56(\mathrm{DT}=1,38)$ & $t(86,4)=2,338^{*}$ \\
\hline Condicionado por la presión de las partes implicadas & $1,81(\mathrm{DT}=1,15)$ & $2,34(\mathrm{DT}=1,40)$ & $t(90,2)=2,597^{*}$ \\
\hline Condicionado por la falta de recursos técnicos & $1,98(\mathrm{DT}=1,13)$ & $2,09(\mathrm{DT}=1,32)$ & $t(106,3)=2,105^{*}$ \\
\hline Condicionado por la dificultad de acceder a fuentes $\bigotimes /$ documentos & $1,89(\mathrm{DT}=1,04)$ & $2,03(\mathrm{DT}=1,17)$ & \\
\hline${ }^{*} p<0,001$ & & & \\
\hline
\end{tabular}

\subsection{Nivel de satisfacción}

En el cuestionario se establecían 11 variables para determinar la satisfacción de los alumnos con las prácticas ${ }^{8}$. Como era de esperar, algunos factores arrojan promedios elevados, en especial los que tienen que ver con las relaciones personales en el seno de la empresa: así, los estudiantes manifiestan un alto grado de satisfacción con los compañeros de redacción o gabinete y con el resto de becarios, oscilando los valores entre 3,72 y 3,99 sobre 5, y siendo un poco más abultados en el caso de la Universidad Complutense que en la de Málaga (tabla 5). El trato dispensado por los superiores merece también puntuaciones por encima de la media (3,32 - UCM- y 3,12 - UMA-), así como la labor desarrollada por el tutor de empresa. Y la carga de trabajo soportada no parece ser motivo de disconformidad, visto en perspectiva, aunque en este caso el índice de la UMA se sitúa tres décimas por debajo del de la UCM $(2,98$ frente a 3,28).

La satisfacción decae ostensiblemente en tres apartados: las oportunidades de promoción, que se perciben como escasas; la remuneración, sin que el hecho de que en la UMA sea por lo general más abultada influya en la opinión de los alumnos; y la labor del tutor académico, desaprobada con rotundidad. En cuanto al promedio global de satisfacción, en la UMA se cifra en 2,83 y en la UCM en 2,98 , sin alcanzar en ningún caso el valor medio de la escala, situado en 3 . Todos estos indicadores, no obstante, pueden matizarse ya que la desviación que presentan resulta estimable.

La prueba t de Student arrojó que UCM y UMA difieren de modo significativo solo en el caso de los horarios de trabajo, luego no cabe establecer aquí distinciones generales basadas en la procedencia de los estudiantes encuestados.

Tabla 5. Índices de satisfacción

\begin{tabular}{|l|l|c|}
\multicolumn{1}{c|}{} & UCM & UMA de Student \\
\cline { 2 - 3 } \multicolumn{1}{c|}{ Satisfacción con los trabajadores de la empresa } & $3,99(\mathrm{DT}=1,18)$ & 3,85 (DT=1,16) \\
\hline Satisfacción con los otros becarios & $3,85(\mathrm{DT}=1,35)$ & $3,72(\mathrm{DT}=1,41)$ \\
\hline Satisfacción con los horarios & $3,57(\mathrm{DT}=1,22)$ & $3,03(\mathrm{DT}=1,36)$ \\
\hline Satisfacción con los superiores & $3,32(\mathrm{DT}=1,31)$ & $3,12(\mathrm{DT}=1,13)$ \\
\hline Satisfacción con su carga de trabajo & $3,28(\mathrm{DT}=1,25)$ & $2,98(\mathrm{DT}=1,31)$ \\
\hline Satisfacción con el tutor de empresa & $3,01(\mathrm{DT}=1,44)$ & 2,97 (DT=1,52) \\
\hline Satisfacción con la estabilidad de su beca & $2,76(\mathrm{DT}=1,45)$ & 2,75 (DT=1,40) \\
\hline Satisfacción con la línea editorial (si procede) & $2,78(\mathrm{DT}=1,28)$ & 2,61 (DT=1,22) \\
\hline Satisfacción con las oportunidades de promocionar & $2,31(\mathrm{DT}=1,33)$ & 2,19 (DT=1,26) \\
\hline Satisfacción con la remuneración & $2,14(\mathrm{DT}=1,27)$ & 2,17 (DT=1,16) \\
\hline Satisfacción con el tutor académico & $1,69(\mathrm{DT}=1,44)$ & 1,69 (DT=1,23) \\
\hline Promedio de satisfacción & $2,98(\mathrm{DT}=0,82)$ & 2,83 (DT=0,84) \\
\hline
\end{tabular}

$* p<0,01$ 
Por otro lado, el análisis de regresión lineal múltiple desveló que en la satisfacción global de los alumnos influyen positivamente la adscripción a la sección de Sociedad, el hecho de poder dar su visión sobre - o incluso decidirel enfoque de los textos, la remuneración percibida y la disponibilidad del tutor cuando se le necesita (tabla 6). Frente a ello y de manera lógica, la firma de piezas con las que no se está de acuerdo por razones éticas o profesionales repercute de manera negativa en el índice de satisfacción global. La regresión permitió explicar en este caso el 32,4\% de la varianza total.

Tabla 6. Coeficientes de regresión para la variable "Satisfacción global con las prácticas"

\begin{tabular}{|c|c|c|c|c|}
\hline \multirow{2}{*}{ Modelo } & \multicolumn{2}{|c|}{ Coeficientes no estandarizados } & \multirow{2}{*}{$\begin{array}{c}\text { Coeficientes } \\
\text { estandarizados }\end{array}$} & \multirow[b]{2}{*}{ Sig. } \\
\hline & B & Error estándar & & \\
\hline Constante & 2,791 & 0,715 & & 0,000 \\
\hline Prácticas en la sección de Sociedad & 0,446 & 0,179 & 0,183 & 0,014 \\
\hline Remuneración & 0,001 & 0,000 & 0,188 & 0,007 \\
\hline Consultas al tutor de empresa & 0,077 & 0,025 & 0,211 & 0,003 \\
\hline Interviene en el enfoque y propone temas & 0,274 & 0,059 & 0,311 & 0,000 \\
\hline Firma algo que le desagrada & $-0,112$ & 0,056 & $-0,129$ & 0,048 \\
\hline Realiza tareas contrarias a la ética & $-0,238$ & 0,076 & $-0,209$ & 0,002 \\
\hline$r^{2}$ ajustado & 0,324 & - & - & - \\
\hline Número de observaciones & 191 & - & - & - \\
\hline$F(26)$ & 4,505 & - & - & 0,000 \\
\hline
\end{tabular}

Para terminar, el cuestionario recoge información sobre las expectativas que las prácticas han generado en los estudiantes (tabla 7). De acuerdo con los datos obtenidos, el deseo de permanecer en la misma empresa resulta mayor entre quienes han trabajado en medios que entre quienes lo han hecho en gabinetes de prensa o similares; y la mayoría de ellos no se plantea cambiar de sector (68,2\% en el caso de la UCM, y 64,1\% en el de la UMA). Los alumnos que han pasado por gabinetes de comunicación se muestran más dubitativos con respecto a su futuro, y también manifiestan en mayor medida la determinación de dedicarse a otra profesión.

Tabla 7. En cinco años...

\begin{tabular}{|l|c|c|c|}
\cline { 2 - 4 } \multicolumn{1}{c|}{} & \multicolumn{2}{c|}{ Medios } & \multicolumn{1}{c|}{ Gabinetes } \\
\cline { 2 - 4 } \multicolumn{1}{c|}{} & UCM & UMA & UCM \\
\hline Quiere quedarse en su empresa & $25,2 \%$ & $12,8 \%$ & $16,7 \%$ \\
\hline Quiere cambiar de empresa, pero no de sector (medios/gabinetes) & $43,0 \%$ & $51,3 \%$ & $19,0 \%$ \\
\hline Quiere cambiar de sector & $6,5 \%$ & $2,6 \%$ & $21,4 \%$ \\
\hline No lo tiene claro & $21,5 \%$ & $25,6 \%$ & $38,1 \%$ \\
\hline Quiere dejar el campo de la comunicación & $3,7 \%$ & $3,7 \%$ & $4,8 \%$ \\
\hline
\end{tabular}

Cabe señalar por último que en ninguna de las variables sometidas a estudio se han detectado divergencias significativas en función del género de los estudiantes.

\section{Conclusiones y discusión}

En esta investigación nos planteábamos abordar aspectos relacionados con las prácticas extracurriculares en Periodismo que hasta ahora no habían sido estudiados en nuestro país, y en determinados casos, tampoco en el ámbito internacional. Lo extenso de la muestra refuerza, creemos, la solidez de las conclusiones alcanzadas, con el añadido de que se ponen en relación por vez primera los programas de prácticas de dos universidades españolas diferentes.

Por lo que respecta a las rutinas profesionales (01), afloran de modo claro dos disfunciones frecuentemente denunciadas en relación con las prácticas: lo prolongado de las jornadas y el elevado volumen de tareas exigido a los estudiantes (Cantalapiedra; Coca; Bezunartea, 2000; Blanco, 2005; Lamuedra, 2007; García-Borrego; Roses; Farias, 2017). Pero un análisis detallado ha permitido identificar factores que condicionan estas dos circunstancias y contribuyen a matizarlas: en primer lugar, el tipo de empresa en que se ubica el alumno (en los gabinetes se trabaja menos horas que en los medios y no se elaboran tantas piezas) y, en segundo lugar, la sección informativa en la que se encuadra, siendo Deportes la que presenta menor carga
Las prácticas se caracterizan por las jornadas prologadas y el elevado volumen de tareas exigido a los alumnos, aunque en los gabinetes de comunicación la carga de trabajo es menor que en los medios 
de trabajo. Nos encontramos por tanto ante un tipo de actividad formativa de naturaleza asimétrica, con niveles de exigencia $-\mathrm{y}$ entendemos que efectos en el aprendizaje- diversos.

Las respuestas al cuestionario ofrecen información detallada sobre cómo se desarrolla el día a día del estudiante en prácticas (en adelante no es posible plantear discusión sobre los resultados puesto que no se han localizado antecedentes en la bibliografía científica disponible). Se identifican instancias que desempeñan una labor socializadora clara desde el punto de vista profesional - los cargos de responsabilidad y el tutor de empresa- y otras cuya implicación es escasa, cuando no nula, caso del tutor académico. El desconocimiento generalizado de esta última figura apunta a una dejación de funciones por parte de la universidad que seguro no ayuda a los alumnos en su proceso de inserción laboral.

Por otro lado, se constata que quienes realizan prácticas gozan de un grado de iniciativa notable, pues tienen la posibilidad de proponer temas y de opinar sobre el enfoque que dan a sus piezas, en lo que sin duda representa una importante contribución a su formación como periodistas.

Asimismo se demuestra la implicación de las empresas en la supervisión de los textos de los estudiantes, que en la mayoría de los casos son sometidos siempre a corrección. Podemos concluir de todo lo anterior que por norma los periodos de prácticas constituyen para los alumnos una experiencia valiosa desde el punto de vista del aprendizaje.

Existen también situaciones poco edificantes en la iniciación al periodismo (O2). Aproximadamente la mitad de los encuestados asegura haber firmado informaciones que colisionaban con sus principios éticos o profesionales, y en uno de cada cinco casos, ni siquiera manifestó su disconformidad. Del mismo modo, en las respuestas de los alumnos afloran algunos condicionantes que han afectado a su trabajo, de carácter sobre todo comercial, vinculados a la productividad o a la necesidad de captar a toda costa la atención del público. El resultado de todo ello es una alienación parcial de los estudiantes, que no logran identificarse con los textos que producen, en lo que constituye la cara menos amable de su socialización profesional.

Dentro de la evaluación global de las prácticas, los aspectos que generan mayor satisfacción entre los encuestados (O3) tienen que ver con las relaciones humanas en el seno de las empresas, tanto horizontales como - más llamativo- verticales. En cuanto a los factores de incidencia negativa, son los esperables (remuneración, expectativas de futuro), con dos salvedades: ni en la UMA ni en la UCM están los alumnos particularmente descontentos con los horarios de trabajo ni con la carga de tareas (lo cual no obsta para que se haya podido vulnerar lo estipulado por los convenios).

Tanto en una universidad como en otra, el índice genérico de satisfacción queda por debajo del aprobado. Parece claro que más allá de su aportación a la formación de los estudiantes, hay margen de mejora en el modo en que se desarrollan las prácticas, si bien existen condiciones de trabajo que ni la tutela académica ni la voluntad de los responsables de las empresas tienen la capacidad de corregir.

La comparativa entre universidades, para terminar, apenas ha ofrecido diferencias significativas desde el punto de vista estadístico (04). Si bien el análisis se ha circunscrito solo a dos instituciones, todo apunta a que en el grado en Periodismo nos encontramos ante un modelo de prácticas extracurriculares geográficamente homogéneo, con la única salvedad del capítulo de remuneración, aunque esto viene condicionado por la rigidez o laxitud de la normativa de cada institución en particular.

\section{Notas}

1. La excepción en este sentido la representa Dinamarca, donde los novice journalists ingresan al mes 2.700 dólares, el equivalente al 60\% del salario medio nacional (Gravengaard; Rimestad, 2014).

2. Especialmente útiles para el diseño del cuestionario resultaron los trabajos de Weaver y Willnat (2012), Gollmitzer (2014), Gravengaard y Rimestad (2014) y Salamon (2015). Lamuedra (2007) y los estudios previos de los autores de este artículo sirvieron de referencia en el plano nacional.

3. La legislación que regula la protección de datos en España impide a las universidades, entre otras cosas, informar de la identidad de los alumnos que realizan prácticas en el marco de sus programas de empleabilidad, de ahí que para realizar esta investigación hubiera que remitir el cuestionario en bloque a estudiantes de distintos cursos.

4. El grado en Periodismo de la Universidad Complutense ofrece en la actualidad 660 plazas para alumnos de nuevo ingreso; en el caso de Málaga, el numerus clausus está fijado en 130.

5. Cifras de estudiantes en prácticas facilitadas por el Servicio de Empleabilidad y Emprendimiento de la Universidad de Málaga y la Oficina de Prácticas y Empleo de la Universidad Complutense.

6. Pese a que las medias obtenidas a través del cuestionario no difieren de manera significativa, UCM y UMA regulan la duración de las jornadas de prácticas de manera diferente. La Complutense (2017) distingue entre el período lectivo, en el cual no pueden exceder las 5 horas diarias o las 25 semanales, y el no lectivo, cuando el tope se sitúa en 7 y 35 horas, respectivamente. En la UMA (2016), en cambio, se establece como preferente una duración no superior a cinco horas diarias o cien al mes. 
7. Por lo que respecta al tamaño de la empresa, su incidencia en las jornadas es creciente a medida que aumenta el número de empleados.

8. El conjunto de variables contempladas resulta altamente fiable para explicar el constructo "índice de satisfacción", ya que arroja un Alfa de Cronbach de 0,856.

\section{Referencias}

APM (2018). Informe anual de la profesión periodística 2018. Madrid: Asociación de la Prensa de Madrid. https://www.apmadrid.es/wp-content/uploads/2019/07/Informe-profesi\%C3\%B3n-2018_baja.pdf

Bjørnsen, Gunn; Hovden, Jan-Fredrik; Ottosen, Rune (2007). “Journalists in the making: Findings from a longitudinal study of Norwegian journalism students". Journalism practice, v. 1, n. 3, pp. 383-403.

https://doi.org/10.1080/17512780701505085

Blanco-Castilla, Elena (2005). “Mujer y poder en los medios: Dificultades para una incorporación plena”. En: APM. Informe anual de la profesión periodística 2005. Madrid: Asociación de la Prensa de Madrid, pp. 99-106. ISBN: 8487641237 https://www.apmadrid.es/wp-content/uploads/2009/02/Informe\%20APM\%202005.pdf

Cantalapiedra, María-José; Coca, César; Bezunartea, Ofa (2000). “La situación profesional y laboral de los periodistas vascos". Zer. Revista de estudios de comunicación, v. 5, n. 9, pp. 169-182.

http://www.ehu.eus/ojs/index.php/Zer/article/view/17441

Fröhlich, Romy; Holtz-Bacha, Christina (eds.) (2003). Journalism education in Europe and North America: An international comparison. Cresskill, New Jersey: Hampton Press. ISBN: 9781572733824

García-Borrego, Manuel; Gómez-Calderón, Bernardo (2018). "Rutinas profesionales y nivel de satisfacción de los estudiantes de Periodismo en prácticas: la brecha entre medios y gabinetes". En: Herrero, Javier; Trenta, Milena (coords.). Comunicación y música: mensajes, manifestaciones y negocios. La Laguna: Universidad de La Laguna, pp. 927-939. ISBN: 9788416458837 http://www.revistalatinacs.org/18SLCS/2018_libro/049_Garcia.pdf

García-Borrego, Manuel; Roses-Campos, Sergio (2016). "Rutinas profesionales de los becarios de Periodismo: el caso de Málaga". Zer. Revista de estudios de comunicación, v. 21, n. 41, pp. 155-169.

http://www.ehu.eus/ojs/index.php/Zer/article/view/17267

García-Borrego, Manuel; Roses-Campos, Sergio; Farias-Batlle, Pedro (2017). “Condiciones de las prácticas profesionales en medios de comunicación: un estudio empírico". Revista latina de comunicación social, n. 72, pp. 430-452. https://doi.org/10.4185/RLCS-2017-1173

Gaunt, Philip (1992). Making the newsmakers: International handbook on journalism training. Westport: Greenwood Press. ISBN: 9780313279843

Gollmitzer, Mirjam (2014). "Precariously employed watchdogs? Perceptions of working conditions among freelancers and interns". Journalism practice, v. 8, n. 6, pp. 826-841.

https://doi.org/10.1080/17512786.2014.882061

Gravengaard, Gitte; Rimestad, Lene (2014). "Socializing journalist trainees in the newsroom: On how to capture the intangible parts of the process". Nordicom review, v. 35, special issue, pp. 81-95.

https://rimestad.dk/onewebmedia/gravengaard_rimestad.pdf

Igartua-Perosanz, Juan-José (2006). Métodos cuantitativos de investigación en comunicación. Barcelona: Bosch. ISBN: 9788497902717

Lamuedra, María (2007). "Estudiantes de periodismo y prácticas profesionales: el reto del aprendizaje". Comunicar, n. 28, pp. 203-211.

https://recyt.fecyt.es/index.php/comunicar/article/view/25995

Lave, Jean; Wenger, Etienne (1991). Situated learning. Legitimate peripheral participation. Cambridge: Cambridge University Press. ISBN: 9780521423748

Ortiz-Sobrino, Miguel-Ángel; Peinado-Miguel, Fernando; Zapata-Palacios, Leila (2014). “Cuáles son las necesidades de los estudiantes de Periodismo para su mejor inserción en el mercado laboral. Estudio del caso de los alumnos de Ciencias de la Información de la UCM que realizan prácticas". En: Actas VI Congreso Internacional Latina de Comunicación Social. Universidad de La Laguna.

http://www.revistalatinacs.org/14SLCS/2014_actas/097_Ortiz.pdf

Pérez-Serrano, María-José; Rodríguez-Barba, Dolores; Rodríguez-Pallares, Miriam (2015). “Mercado de la comunicación y estudiantes de Periodismo. Estructura de la demanda de perfiles profesionales". Revista latina de comunicación social, n. 70, pp. 209-229. 
https://doi.org/10.4185/RLCS-2015-1043

Preston, Paschal (2009). Making the news: Journalism and news cultures in Europe. Londres: Routledge. ISBN: 9780 415461894

Salamon, Errol (2015). "(De)valuing intern labour: Journalism internship pay rates and collective representation in Canada". Triple C. Communication, capitalism \& critique, v. 13, n. 2, pp. 438-458.

https://doi.org/10.31269/triplec.v13i2.573

Segarra-Saavedra, Jesús; Páramo-Galdón, Patricia; Puebla-Martínez, Belén (2012): “Jóvenes emprendedores y sus proyectos de comunicación en la red: estudio de caso de Periodistas en potencia y Portal del sur". adComunica, n. 4, pp. 39-61.

http://www.adcomunicarevista.com/ojs/index.php/adcomunica/article/view/68/96

Splichal, Slavko; Sparks, Colin (1994). Journalists for the $21^{\text {st }}$ century: Tendencies of professionalization among first-year students in 22 countries. Norwood: Ablex Publishing. ISBN: 9780893919542

Standing, Guy (2011). The precariat: The new dangerous class. Londres: Bloomsbury Academic. ISBN: 9781474294164

UCM (2017). Reglamento de prácticas académicas externas de la Universidad Complutense de Madrid. Universidad Complutense de Madrid.

https://bit.ly/2FQzCWU

UMA (2013). Normativa de prácticas externas de la Universidad de Málaga. Universidad de Málaga.

https://bit.ly/2NicpMm

Weaver, David H.; Willnat, Lars (eds.) (2012). The global journalist in the $21^{\text {st }}$ century. Londres: Routledge, pp. $220-233$. ISBN: 9780415885775

Willig, Ida (2016). "We all think the same". Internships, craft and conservation”. En: Hovden, Jan-Fredrik; Nygren, Gunnar; Zilliacus-Tikkanen, Henrika. Becoming a journalist. Journalism education in the Nordic countries. Gotenburg: Nordicom, pp. 39-50. ISBN: 9789187957345

https://www.nordicom.gu.se/sites/default/files/publikationer-hela-pdf/becoming_a_journalist.pdf

\section{Inforảrea}

Ayudamos a tu organización en la transformación digital yel gobierno de la información

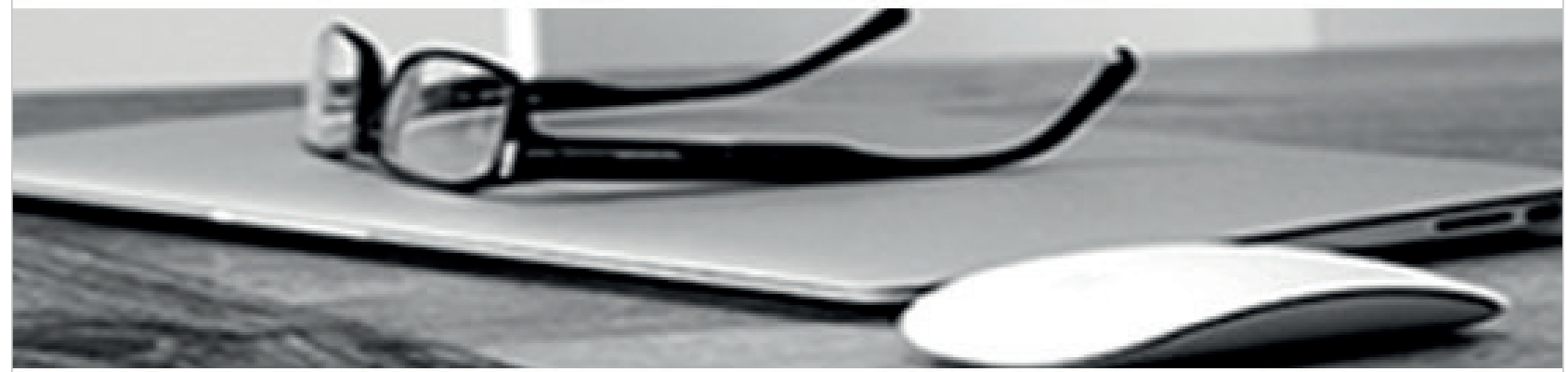

* Consultoría estratégica en gestión y gobierno de la información

* Gestión documental y "records management"

* Gestión de contenidos, intranets corporativas y entornos de colaboración

* Estudios especializados

Clientes satisfechos, cientos de empresas nacionales e internacionales y más de 30 años de experiencia son la mejor garantía de nuestra reputación. 